\title{
Primary ciliary dyskinesia: current state of the art
}

\section{Andrew Bush, Rahul Chodhari, Nicola Collins, Fiona Copeland, Pippa Hall, Jonny Harcourt, Mohamed Hariri, Claire Hogg, Jane Lucas, Hannah M Mitchison, Christopher O'Callaghan, Gill Phillips}

Arch Dis Child 2007;92:1136-1140. doi: 10.1136/adc.2006.096958

Primary ciliary dyskinesia (PCD) is usually inherited as an autosomal recessive disorder and presents with upper and lower respiratory tract infection, and mirror image arrangement in around $50 \%$ of cases. Cilia dysfunction is also implicated in a wider spectrum of disease, including polycystic liver and kidney disease, central nervous system problems including retinopathy and hydrocephalus, and biliary atresia. Cilia are complex structures, containing more than 250 proteins; recent studies have begun to locate PCD genes scattered throughout the genome. Screening tests for PCD include nasal nitric oxide and in vivo tests of ciliary motility such as the saccharin test. Specific diagnosis requires examination of cilia by light and electron microscopy, with epithelial culture in doubiful cases. This is only available in supra-regional centres, recently centrally funded by the National Commissioning Group. Treatment is not evidence based and recommendations are largely extrapolated from cystic fibrosis and other suppurative lung diseases.

See end of article for authors' affiliations

Correspondence to: Andrew Bush, Department of Paediatric Respiratory Medicine, Royal Brompton Hospital, Sydney Street, London SW3 6NP, UK. a.bush@rbh.nthames.nhs. uk

Accepted 25 June 2007 Published Online First 30 July 2007
$\mathrm{P}$ rimary ciliary dyskinesia (PCD) is usually an autosomal recessive disease characterised by chronic upper and lower respiratory tract infection, and in nearly 50\% cases, mirror image arrangement. ${ }^{1}$ The triad of mirror image arrangement, bronchiectasis and sinusitis is known as Kartagener syndrome. It is caused by a congenital reduction or absence of ciliary function. PCD must be distinguished from the numerous causes of secondary ciliary dysfunction. PCD is often diagnosed late, ${ }^{2}$ after a period of chronic ill health. Cilia are also implicated in polycystic hepatic and renal disease, hydrocephalus, biliary atresia, retinal degeneration and rare syndromes. ${ }^{2}{ }^{3}$ Motile nodal cilia are responsible for normal right-left orientation, but there may be other important developmental roles elsewhere in the embryo. ${ }^{3}$ This article provides an update on PCD, which is particularly timely as it coincides with the development of a diagnostic screening service for PCD in England. The National Commissioning Group (NCG) is funding centres in London, Leicester and Southampton to utilise state of the art diagnostic methods to increase the numbers of patients diagnosed. Since the test is centrally funded, the referring centre incurs no costs. Early diagnosis of PCD is likely to have a significant effect on both short-term and long-term morbidity, ${ }^{56}$ although this cannot be proven by any practical study. The development of proper diagnostic services will also lead to the establishment of a national registry, which should facilitate the performance of randomised controlled trials.

\section{BIOPHYSICS OF CILIA}

Over 250 proteins make up the classical ciliary axoneme that forms a " $9+2$ " arrangement where nine peripheral doublets surround the central microtubular pair ${ }^{7}$ (fig 1). The ATPase activity of the dynein arms generates the force required for ciliary beating and bending. Situs inversus is seen in up to $50 \%$ of patients with PCD due to abnormal movement of nodal cilia during embryogenesis.

\section{GENETICS}

PCD has an estimated prevalence of 1:15-30 000 live births, although this is likely be an underestimate as underdiagnosis is common. ${ }^{1}$ PCD is usually an autosomal recessive disorder, however occasional instances of X-linked transmission have been reported..$^{7}$ The molecular complexity of the axonemes of cilia and sperm means that there are a large number of potential candidate genes. PCD is genetically heterogeneous, being caused by mutations in a number of different genes. ${ }^{9-11}$ So far two genes encoding dyneins have been confirmed to cause PCD. The DNAH5 gene located on chromosome 5p15.2 encodes a heavy chain dynein and the DNAIl gene on 9p13.3 encodes a dynein intermediate chain. Two additional dyneins have been associated with PCD. Homozygous mutations in the DNAHIl gene on chromosome 7, which encodes an axonemal heavy chain, were determined in an individual with situs inversus but no apparent ciliary ultrastructural defects. ${ }^{12}$ Proteomic studies have also implicated DNAH7, another dynein heavy chain. In ciliated epithelial cells from an individual with PCD and an ultrastructural defect of absent inner arms, the DNAH7 protein was found to be expressed but not assembled into the cilia. No mutations in the DNAH7 gene were detected, so the possibility that this may be an indirect effect arising from mutations in another gene could not be excluded.

Genetic linkage studies have found significant evidence for several other PCD loci, notably those on chromosomes 15q13-15, 15q24-q25, 16p and $19 q$, and support for an additional chromosome 1 locus. ${ }^{10}{ }^{13}{ }^{14}$ Although these loci have been mapped, the causative gene remains to be determined. A number of candidate genes for PCD with

Abbreviations: $\mathrm{ACBT}$, active cycle of breathing technique; CF, cystic fibrosis; NCG, National Commissioning Group; $\mathrm{NO}$, nitric oxide; OME, otitis media with effusion; $P C D$, primary ciliary dyskinesia; PEP, positive expiratory pressure 

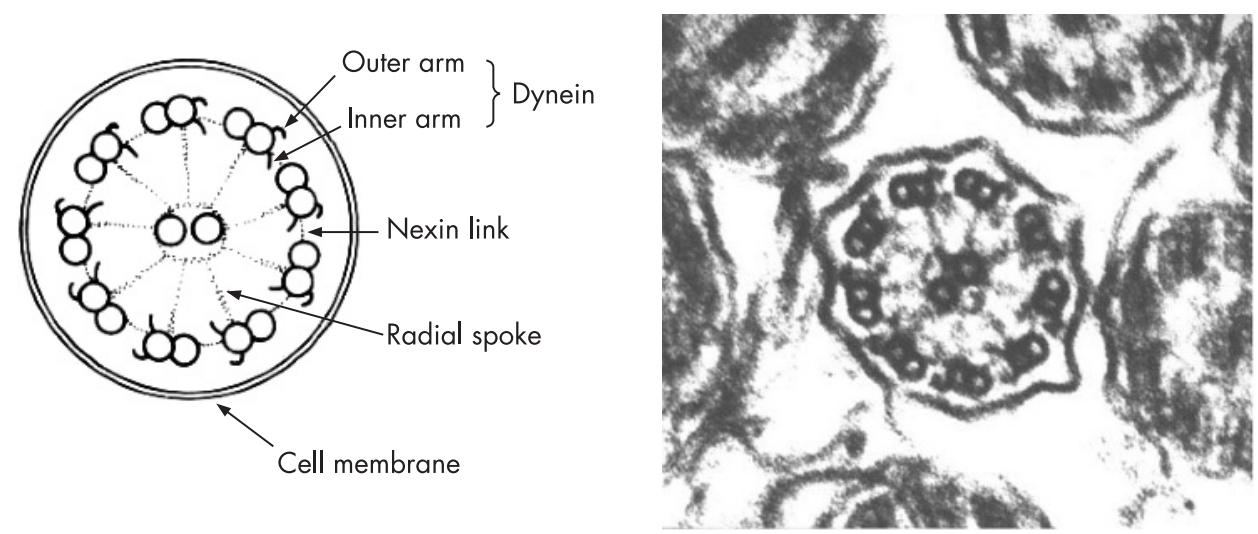

Figure 1 Schematic diagram of the basic structure of cilia.

suggestive roles in ciliogenesis have been selected for mutation screening in PCD patients but excluded from playing a role in the disease. ${ }^{9}$

Certain features of PCD such as situs inversus may be observed in other disorders that are more closely associated with dysfunction of the non-motile or sensory cilia. Sensory cilia are more ubiquitous than motile cilia, being found in most tissues of the body including in the kidney, retina and embryonic node, and their dysfunction results in a broad range of phenotypes including renal and retinal problems. ${ }^{4}$ This putative connection is currently clearest for retinal function, since several cases are known of individuals who have features consistent with PCD in addition to retinitis pigmentosa or to Usher syndrome. ${ }^{815}$ Mutations in the X-linked retinitis pigmentosa GTPase regulator gene $(R P G R)$ gene have recently been identified in two brothers with PCD and retinitis pigmentosa, ${ }^{15}$ and there are a number of other RPGR mutations. $^{8}$

\section{WHEN TO SUSPECT THE DIAGNOSIS OF PCD}

PCD may be suspected because of respiratory disease or mirror image arrangement (conventional diagnostic clues) or because a non-respiratory condition such as biliary atresia has been diagnosed (associated diagnosis).

Diagnosis is frequently made late, ${ }^{2}$ in part because it presents with symptoms (rhinitis, secretory otitis media, cough) which are common in children. ${ }^{16}$ General paediatricians must be alert to the condition and take a careful, focussed history. Although there is no proven evidence that early diagnosis is beneficial, in one series, bronchiectasis at diagnosis was only seen in those diagnosed over 4 years of age, ${ }^{2}$ and in a second series, lung function at diagnosis was significantly worse in those diagnosed in adult life. ${ }^{5}$ This is at least supportive evidence that early diagnosis is beneficial.

There are no data to weight the significance of particular symptoms or construct receiver operating curves, but the common diagnostic features of PCD are listed in table 1 as a guide. A combination of upper and lower airway symptoms is usual.

\section{Conventional diagnostic clues \\ Antenatal presentation}

Heterotaxy (usually mirror image organ arrangement) on antenatal ultrasound scanning should lead to consideration of the diagnosis, although most babies with mirror image arrangement do not have PCD.

\section{Presentation in the newborn period}

- Continuous rhinorrhoea from the first day of life; this is highly likely to be due to PCD
- Respiratory distress or neonatal pneumonia with no obvious predisposing cause

- Mirror image arrangement (but see "Antenatal presentation" above)

- Diagnosis by screening because of a positive family history

\section{Presentation in childhood}

- Chronic productive or "wet" cough. Clearly, other causes such as cystic fibrosis (CF) will likely need to be ruled out first by appropriate testing, before referral for ciliary function, unless the child also has mirror image arrangement or other features suggestive of PCD. Isolated dry cough is not likely to be due to PCD.

- Atypical "asthma", non-responsive to treatment, especially if a wet-sounding cough is present

- "Idiopathic" bronchiectasis. Diagnosis of bronchiectasis by screening because of a positive family history of PCD. This accounted for $10 \%$ of cases in one series. ${ }^{2}$

- Rhinosinusitis. Daily rhinitis is typical, without remission, and sometimes in older children severe sinusitis despite multiple surgical procedures; nasal polyps are rare, and more commonly due to CF.

- Otitis media with effusion (OME). Typically, if tympanostomy (ventilation tubes) are inserted, there is usually a prolonged smelly ear discharge for weeks not responding to treatment and with no improvement in hearing.

\section{Presentation in adolescence and adult life}

- As above, for childhood

- Ectopic pregnancy and subfertility in women ${ }^{6}{ }^{17}$

- Male infertility (immotile sperm, but 50\% of PCD males are fertile, ${ }^{18}$ see below)

\section{PCD as an associated diagnosis}

In addition to the above, PCD should be at least considered when the following diagnoses are made, in particular if there is a family history of more than one of these conditions, or if the patient has other features of PCD. ${ }^{3}$

- Complex congenital heart disease, especially with disorders of laterality ${ }^{19}$

- Polycystic kidney and liver disease

- Hydrocephalus

- Biliary atresia

- Severe oesophageal disease (oesophageal atresia, severe reflux $)^{19}$

- Retinal degeneration, including retinitis pigmentosa 
Table 1 Features leading to the diagnosis of PCD

\begin{tabular}{ll}
\hline Presenting feature & $\begin{array}{l}\text { Number }(\%) \text { of } \\
\text { cases }(\mathbf{n}=55)\end{array}$ \\
\hline Significant neonatal respiratory distress & $37(67)$ \\
Abnormal situs & $38(69)$ \\
Cough most days & $46(84)$ \\
Sputum production & $24(44)$ \\
Rhinorrhoea from the newborn period & $42(76)$ \\
Wheeze & $16(29)$ \\
Sinusitis & $6(11)$ \\
Serous otitis media & $28(51)$ \\
Hearing loss & $14(25)$ \\
Diagnosis made in a sibling & $6(11)$ \\
\hline
\end{tabular}

Modified from Coren et al. ${ }^{2}$ Most children had multiple symptoms, typically a combination of upper and lower airway disease.

\section{MAKING THE DIAGNOSIS \\ Eliminating other conditions}

Although a child with absolutely typical features of PCD will appropriately be referred directly for ciliary function studies, in most cases testing for commoner conditions which enter the differential diagnosis will precede referral. The nature of the tests carried out will be determined by the individual clinical scenario.

\section{Screening for PCD}

The most popular screening tests for PCD are the saccharin test and the measurement of nasal nitric oxide (NO). The saccharin test involves placing a microtablet of saccharin on the inferior turbinate and recording the time taken for the subject to taste it. It is difficult to perform and can be unreliable in children. ${ }^{20}$ An abnormal result must be confirmed by further tests (see below). In PCD nasal and exhaled NO is low ${ }^{21} 22$ for reasons that are still unclear and measurement of nasal NO, which is more discriminatory than exhaled $\mathrm{NO}$, is a good screening test. ${ }^{23}$ However, as there is occasional overlap with other respiratory conditions such as CF, confirmation of the diagnosis of PCD will always require further testing. ${ }^{1624}$

\section{Diagnostic testing}

A combined approach to the diagnostic testing of suspected PCD reduces the number of cases that are incorrectly diagnosed. Tests include:

- ciliary beat frequency measurement,

- ciliary beat pattern analysis,

- electron microscopy of ciliary ultrastructure,

- measurement of ciliary disorientation, and

- in difficult cases, cell culture with re-growth of the ciliated epithelium.

Strips of ciliated usually nasal epithelium obtained by brushing the nose are used to assess ciliary structure and function. The nasal brushing is an out-patient procedure which only takes a few seconds. The brushing causes minor discomfort, and very occasionally, minor epistaxis. The sample may be taken by an appropriately trained paediatrician in a general hospital, and sent by courier to the NCG centre, by prior arrangement. Assessment of beat pattern by slow motion analysis using high-speed video photography in conjunction with beat frequency measurements has been shown to identify defects that may have been missed by analysis of beat frequency alone..$^{25}$ An extensive range of normal values for children and adults using high-speed video analysis has now been published. ${ }^{26}$

Some groups have diagnosed PCD in patients who had clinical symptoms consistent with PCD and a slow ciliary beat frequency despite a normal ciliary ultrastructure. ${ }^{27}$ It is still to be determined whether such patients have an inherited defect affecting function that cannot be seen on electron microscopy. While such patients receive clinical treatment similar to that for PCD, they cannot be confidently diagnosed as having PCD.

Examination of ciliary ultrastructure remains the definitive test for PCD. There are a number of ultrastructural phenotypes that can cause PCD. ${ }^{28}$ Most cases of PCD are due to a lack of outer dynein arms or a combined lack of both inner and outer dynein arms. Other defects include isolated lack of inner dynein arms or lack of inner dynein arms combined with a radial spoke defect. Rarer defects include those of the central microtubular pair, such as transposition or central microtubular agenesis. ${ }^{29}$

On occasions the diagnosis of PCD may be difficult due to damage to the respiratory epithelium secondary to infection or inflammation. If secondary damage is still present making the diagnosis difficult or if the patient is suspected of having an unusual PCD phenotype, ciliated cells are cultured. Secondary damage is virtually absent after ciliogenesis in a suspension culture. $^{30}$ This technique is only necessary in cases of doubt and is not mandatory for the diagnosis of clear-cut cases of PCD.

Overall, around $10 \%$ of initial tests are equivocal. A second brushing reduces the figure to $5 \%$ (unpublished data). In the absence of another gold standard test, it is impossible to calculate false positive and false negative rates. The most important cause of false positive tests are post-viral changes, and if there is any doubt, the tests should be repeated before confirming the diagnosis.

\section{RESPIRATORY MANAGEMENT}

Appropriate medical therapy has been shown to prevent deterioration in lung function. ${ }^{56}$ The optimal balance of care between the regional centre and district hospital has not been determined, and is likely in practice to be driven by geography and the facilities available locally. If the family live reasonably near a centre with special expertise in PCD, a shared care arrangement, analogous to CF clinics, might be appropriate. They should be looked after only in a setting where multidisciplinary review is available. Management is not evidence based. Respiratory management consists of:

- regular respiratory monitoring,

- airway clearance by combinations of physiotherapy and physical exercise, and

- aggressive treatment of upper and lower airways infections.

\section{Respiratory monitoring}

Recommendations are based on CF clinic practices. In some centres, specialist PCD clinics which include respiratory, ENT and general care at the one visit, have been set up. In addition to general paediatric care, monitoring should include pulse oximetry and age appropriate lung function tests. Regular sputum or cough swab cultures should be performed. ${ }^{1}$ Chest radiographs are probably relatively insensitive. High resolution computed tomography of the lungs is used to define the extent of bronchiectasis but is used only very infrequently to monitor disease progression.

\section{Medical interventions}

PCD lung disease is progressive if not adequately treated, and early therapeutic interventions result in better symptom control. ${ }^{31}$ The common infecting organisms in children are Haemophilus influenza and Staphylococcus aureus, but Streptococcus pneumoniae, Pseudomonas aeruginosa and non-tuberculous mycobacteria have also been reported, although these are usually a feature of disease in adults. ${ }^{6}$ There is no evidence to recommend or otherwise the use of prophylactic antibiotics. If repeated 
courses of oral antibiotics are required, prophylaxis should be considered. High-dose oral antibiotics should be given at the first sign of worsening respiratory symptoms or deterioration in lung function. Where possible, antibiotics should be chosen on the basis of sputum or cough swab culture and sensitivity. Occasionally, broncho-alveolar lavage may be necessary to obtain adequate specimens in non-sputum producing children.

If $P$ aeruginosa is isolated, most use an eradication regime similar to CF. For established chronic infection with this organism, long-term nebulised anti-pseudomonal antibiotics are considered. If symptoms do not respond to oral antibiotics, then intravenous therapy is given. A regular programme of 3monthly intravenous therapy should be considered in patients who are not doing well.

Regular bronchodilators are not particularly effective. No trials have studied any anti-inflammatory therapy. Anecdotally, many of these patients have previously been misdiagnosed as having asthma and given asthma therapy, which is ineffective. It is very important to be proactive in discontinuing medications which have been started in error and which are not effective. The use of rhDNAse is unproven; anecdotally some patients show an improvement. Nebulised saline may be effective in augmenting mucus clearance.

PCD patients should receive all childhood immunisations, including influenza A and pneumococcal. Complications of bronchiectasis and chronic lung disease become more prominent with age. ${ }^{6}$ Lobectomy for bronchiectasis is rarely recommended. A few patients require lung transplantation. Overall, the medical therapy of PCD is more successful than that for CF; once treatment is instituted, even if there is markedly impaired lung function at diagnosis, PCD should be stabilised. ${ }^{5} 6$

\section{AIRWAY INFLAMMATION}

There has been little work on airway inflammation in PCD. Examination of spontaneously expectorated sputum reveals predominantly neutrophilic cytology, as in CF. ${ }^{32}$ Since CF is characterised by relentless deterioration, and PCD by stability, one might predict that the inflammatory response would be greater in CF, but this is not the case; mucus biophysical and transport properties are the same, and sputum interleukin (IL)8 is actually three times higher in PCD. ${ }^{33}$

\section{PHYSIOTHERAPY}

There is no evidence that physiotherapy treatment is beneficial in PCD and options should be tailored to the individual. The parents of babies and infants should be shown gravity assisted positioning and intermittent chest percussion; the optimum duration and frequency is not known. Jumping and blowing games should be encouraged early on and replaced by the active cycle of breathing technique (ACBT) as the child becomes older. ${ }^{34}$ Independence is encouraged in older patients. Alternative techniques include the PEP (positive expiratory pressure) mask or mouthpiece ${ }^{35}$ and oscillating PEP devices such as the Flutter ${ }^{36}$ or Acapella. ${ }^{37}$ Adherence to treatment must also be considered. ${ }^{31}$

Physical exercise may help sputum clearance. Exercise has been shown to be a better bronchodilator than the use of a $\beta-2$ agonist in PCD. ${ }^{38}$ Exercise should be encouraged from early on. The role of inspiratory muscle training in PCD is not known. ${ }^{39}$

\section{ENT MANAGEMENT}

Abnormal mucociliary clearance within the upper airway in PCD leads to OME and chronic mucus pooling within the nasal cavity and paranasal sinuses. OME, often severe, is universal among infants with PCD and resolves spontaneously at around age $13 .^{40}$ It presents with either hearing loss and speech delay or as recurrent acute otitis media. Ventilation tube insertion is associated with a high level of post-operative mucoid otorrhoea and tympanic membrane perforation. ${ }^{41}$ This operation should be avoided if at all possible, and certainly should not be performed before consultation with a surgeon familiar with the management of PCD. The best way to treat otorrhoea is by cleaning the ear, avoiding water in the ear, and administering topical non-ototoxic anti-pseudomonal antibiotic eardrops, for example ciprofloxacin. All children with PCD should have regular hearing tests at least until adolescence. If necessary, hearing aids are recommended until spontaneous resolution of hearing loss. For milder hearing loss, educational support may be all that is needed. Speech delay should be promptly managed with speech therapy.

Chronic mucoid rhinorrhoea may be treated using saline nasal douches. Long-term topical nasal steroids are of uncertain benefit for rhinorrhoea but will treat any degree of rhinosinusitis. Recurrent acute or chronic sinusitis is rare. It may be managed with prolonged systemic antibiotics but occasionally requires endoscopic sinus surgery. Nasal polyps are virtually never seen. Obstructive sleep apnoea secondary to adenotonsillar hypertrophy is also not a typical association with PCD.

\section{OTHER SYSTEMS}

Around half of the men who have PCD are infertile, although many men have fathered children with no medical intervention. ${ }^{18}$ Infertility in men with PCD is entirely attributed to the poor motility of the sperm. There are several reports of the birth of healthy children after intracytoplasmic sperm injection. Females have reduced fertility with higher risk for ectopic pregnancy. ${ }^{17}$ Rarer associations of PCD are described above. The management of these associated conditions is unaffected by coincident PCD, except in so far as respiratory issues must be remembered if a general anaesthetic is required.

\section{NURSING PERSPECTIVE}

Very little has been published on the role of the paediatric nurse specialist in the care of children with PCD, and the models of care have to be based on the CF nurse specialist. The nurse should be involved in pretest counselling. After diagnosis, the psychological and practical support provided by the respiratory nurse includes clinical management, education, social care and counselling. ${ }^{42}$ The nurse specialist is ideally placed to become the key worker, providing a link between hospital, home, the school and primary care. ${ }^{43}$ Children with PCD must be encouraged to lead a normal life and should be encouraged to participate in all usual activities at home and school including sport.

\section{SUMMARY AND CONCLUSIONS}

Ciliopathy has now become recognised as a multisystem disease, of which PCD is an important subgroup. The current challenge for paediatricians is to increase diagnostic awareness, and make early and appropriate referrals to the new diagnostic services before the child has sustained lung damage. Next, we need to find ways through multicentre collaborations of increasing the evidence base for treatment. In the future, basic science may open the way for curative treatment, as opposed to mere management of symptoms. However, none of this will happen unless as a routine, a properly focused respiratory history is taken, and the diagnosis of PCD considered outside the context of specialist clinics.

\section{Authors' affiliations Andrew Bush, Nicola Collins, Pippa Hall, Claire Hogg, Royal Brompton Hospital, London, UK \\ Rahul Chodhari, Great Ormond Street Hospital, London, UK}


Fiona Copeland, Primary Ciliary Dyskinesia Family Support Group, UK Jonny Harcourt, Chelsea and Westminster Hospital, London, UK Mohamed Hariri, Charing Cross Hospital, London, UK

Jane Lucas, Chest Medicine, Southampton University Hospitals NHS Trust, Southampton, UK

Hannah M Mitchison, General and Adolescent Paediatric Unit, UCL Institute of Child Health, London, UK

Christopher O'Callaghan, Leicester Royal Infirmary and University of Leicester, Leicester, UK

Gill Phillips, Faculty of Health, Leeds Metropolitan University, Leeds, UK

Competing interests: None declared.

\section{REFERENCES}

1 Bush A, Cole P, Hariri M, et al. Primary ciliary dyskinesia: diagnosis and standards of care. Eur Respir J 1998;12:982-8.

2 Coren ME, Meeks M, Buchdahl RM, et al. Primary ciliary dyskinesia (PCD) in children - age at diagnosis and symptom history. Acta Paediatr 2002;91:667-9.

3 Ong ACM, Wheatley DN. Polycystic kidney disease: the ciliary connection. Lancet 2003:361:774-6.

4 Badano JL, Mitsuma N, Beales PL, et al. The ciliopathies: an emerging class of human genetic disorders. Annu Rev Genomics Hum Genet 2006;7:125-48.

5 Ellerman A, Bisgaard $\mathrm{H}$. Longitudinal study of lung function in a cohort of primary ciliary dyskinesia. Eur Respir J 1997; 10:2376-9.

6 Noone PG, Leigh MW, Sannuti A, et al. Primary ciliary dyskinesia: diagnostic and phenotypic features. Am J Respir Crit Care Med 2004;169:459-67.

7 Narayan D, Krishnan SN, Upender M, et al. Unusual inheritance of primary ciliary dyskinesia (Kartagener's syndrome). J Med Genet 1994;31:493-6.

8 lannaccone A, Brever DK, Wang XF, et al. Clinical and immunohistochemical evidence for an $\mathrm{X}$ linked retinitis pigmentosa syndrome with recurrent infections and hearing loss in association with an RPGR mutation. J Med Genet 2003;40:e118.

9 Geremek M, Witt M. Primary ciliary dyskinesia: genes, candidate genes and chromosomal regions. J Appl Genet 2004;45:347-61.

10 Chodhari R, Mitchison HM, Meeks M. Cilia, primary ciliary dyskinesia and molecular genetics. Paediatr Respir Rev 2004;5:69-76.

11 Blouin JL, Meeks M, Radhakrishna U. Primary ciliary dyskinesia: a genome-wide linkage analysis reveals extensive locus heterogeneity. Eur J Hum Genet 2000;8:109-18.

12 Bartoloni L, Blouin JL, Pan Y, et al. Mutations in the DNAH1 1 (axonemal heavy chain dynein type 11) gene cause one form of situs inversus totalis and most likely primary ciliary dyskinesia. Proc Natl Acad Sci U S A 2002;99:10282-6.

13 Geremek M, Zietkiewicz E, Dieh SR, et al. Linkage analysis localises a Kartagener syndrome gene to a $3.5 \mathrm{cM}$ region on chromosome 15q24-25. J Med Genet 2006;43:e1.

14 Gutierrez-Roelens I, Sluysmans T, Jorissen $M$, et al. Localization of candidate regions for a novel gene for Kartagener syndrome. Eur J Hum Genet 2006;14:809-15.

15 Bonneau D, Raymond F, Kremer C, et al. Usher syndrome type I associated with bronchiectasis and immotile nasal cilia in two brothers. J Med Genet 1993:30:253-4.

16 Bush A, O'Callaghan C. Primary ciliary dyskinesia. Arch Dis Child 2002;87:363-5.

17 Halbert SA, Patton DL, Zarutskie PW, et al. Function and structure of cilia in the fallopian tube of an infertile woman with Kartagener's syndrome. Hum Reprod 1997;12:55-8.

18 Munro NC, Currie DC, Lindsay KS, et al. Fertility in men with primary ciliary dyskinesia presenting with respiratory infection. Thorax 1994;49:684-7.
19 Gemou-Engesaeth V, Warner JO, Bush A. New associations of primary ciliary dyskinesia syndrome. Pediatr Pulmonol 1993;16:9-12.

20 Canciani M, Barlocco EG, Mastella G, et al. The saccharin method for testing mucociliary function in patients suspected of having primary ciliary dyskinesia. Pediatr Pulmonol 1988;5:210-14.

21 Lundberg JO, Weitzberg E, Nordvall SL, et al. Primarily nasal origin of exhaled nitric oxide and absence in Kartagener's syndrome. Eur Respir J 1994; 7:1501-4.

22 Karadag B, James AJ, Gultekin E, et al. Nasal and lower airway level of nitric oxide in children with primary ciliary dyskinesia. Eur Respir J 1999;13:1402-5.

23 Narang I, Ersu R, Wilson NM, et al. Nitric oxide in chronic airway inflammation in children: diagnostic use and pathophysiological significance. Thorax 2002;57:586-9.

24 Balfour-Lynn IM, Laverty A, Dinwiddie R. Reduced upper airway nitric oxide in cystic fibrosis. Arch Dis Child 1996;75:319-22.

25 Chilvers MA, Rutman A, O'Callaghan C. Ciliary beat pattern is associated with specific ultrastructural defects in primary ciliary dyskinesia. J Allergy Clin Immunol 2003;112:518-24

26 Chilvers M, Rutman A, O'Callaghan C. Functional analysis of cilia and ciliated epithelial ultrastructure in healthy children and young adults. Thorax 2003;58:333-8.

27 Buchdahl RM, Reiser J, Ingram D, et al. Ciliary abnormalities in respiratory disease. Arch Dis Child 1988;63:238-43.

28 Afzelius BA. Cilia-related diseases. J Pathol 2004;204:470-7.

29 Stannard W, Rutman A, Wallis C, et al. Central microtubular agenesis causing primary ciliary dyskinesia. Am J Respir Crit Care Med 2004;169:634-7.

30 Jorissen M, Willems T, Van der Schueren B, et al. Ultrastructural expression of primary ciliary dyskinesia after ciliogenesis in culture. Acta Otorhinolaryngol Belg 2000;54:343-56.

31 McManus IC, Mitchison HM, Chung EM, et al. Primary ciliary dyskinesia (Siewert's/Kartagener's syndrome): respiratory symptoms and psycho-social impact. BMC Pulm Med 2003;3:4.

32 Zihlif N, Paraskakis E, Lex C, et al. Correlation between cough frequency and airway inflammation in children with primary ciliary dyskinesia. Pediatr Pulmonol 2005;39:551-7.

33 Bush A, Payne D, Pike S, et al. Mucus properties in children with primary ciliary dyskinesia: comparison with cystic fibrosis. Chest 2006;129:118-23.

34 Pryor J, Prasad A. Physiotherapy for respiratory and cardiac problems: adults and paediatrics, 3rd edn. London: Churchill Livingstone, 2003.

35 Gremmo M, Guenza M. Positive expiratory pressure in the physiotherapeutic management of primary ciliary dyskinesia in paediatric age. Monaldi Arch Chest Dis 1999;54:255-7

36 Thompson C, Harrison S, Ashley J, et al. Randomised crossover study of the Flutter device and the active cycle of breathing technique in non-cystic fibrosis bronchiectasis. Thorax 2002;57:446-8.

37 Volsko T, DiFiore J, Chatburn R. Performance comparison of two oscillating positive expiratory pressure devices: Acapella versus Flutter. Respir Care 2003;48:124-30.

38 Phillips G, Thomas S, Heather S, et al. Airway response of children with primary ciliary dyskinesia to exercise and B2-agonist challenge. Eur Respir J 1998;11:1389-91.

39 Bradley J, Moran F, Greenstone M. Physical training for bronchiectasis. Cochrane Database Syst Rev 2002;3:CD002166.

40 Majitha A, Fong J, Hariri $M$, et al. Hearing outcomes in children with primary ciliary dyskinesia - a longitudinal study. Int J Pediatr Otorhinolaryngol 2005;69:1061-4

41 Hadfield PJ, Rowe-Jones J, Bush A, et al. Treatment of otitis media with effusion in children with primary ciliary dyskinesia. Clin Otolaryngol 1997:22:302-6.

42 UK Cystic Fibrosis Nurse Specialist Group. National consensus standards for the nursing management of cystic fibrosis. Bromley: CF Trust, 2001.

43 Dyer J. Cystic fibrosis: role of the nurse specialist in a life long condition. Airways J 2003;1:198-201. 